\title{
The topological long range order in QCD. Applications to heavy ion collisions and cosmology.
}

\author{
Ariel R. Zhitnitsky ${ }^{1, a}$ \\ ${ }^{1}$ Department of Physics and Astronomy, University of British Columbia, Vancouver, B.C. V6T 1Z1, Canada
}

\begin{abstract}
We argue that the local violation of P invariance in heavy ion collisions is a consequence of the long range topological order which is inherent feature of strongly coupled QCD. A similar phenomenon is known to occur in some topologically ordered condensed matter systems with a gap. We also discuss possible cosmological applications of this long range order in strongly coupled gauge theories. In particular, we argue that the de Sitter behaviour might be dynamically generated as a result of the long range order. In this framework the inflaton is an auxiliary field which effectively describes the dynamics of topological sectors in a gauge theory in the expanding universe, rather than a new dynamical degree of freedom.
\end{abstract}

\section{Introduction}

Recently it has become clear that quantum anomalies play very important role in the macroscopic dynamics of relativistic fluids. Much of this progress is motivated by very interesting ongoing experiments on local $\mathcal{P}$ and $C \mathcal{P}$ violation in QCD as studied at RHIC [1-3] and, more recently, at the LHC [4-7]. It is likely that the observed asymmetry is due to charge separation effect [8] as a result of the chiral anomaly, see recent review [9] as an introduction to the subject. The ideas formulated in [8] were further developed in follow up papers $[10,11]$ where the effect was coined as chiral magnetic effect (CME).

We shall not discuss a number of subtle questions of the CME in the present work by referring to a recent review [9]. Instead, we concentrate on a single crucial element for CME to be operational. Namely, the key assumption of the proposal $[8,9]$ is that the region where the so-called $\left\langle\theta(\vec{x}, t)_{\text {ind }}\right\rangle \neq 0$ should be much larger in size than the scale of conventional QCD fluctuations which have typical correlation lengths of order $\sim \Lambda_{O C D}^{-1}$. The $\theta(\vec{x}, t)_{\text {ind }}$ parameter enters the effective lagrangian as follows, $\mathcal{L}_{\theta}=-\theta_{\text {ind }} q$ where $q \equiv \frac{g^{2}}{64 \pi^{2}} \epsilon_{\mu \nu \rho \sigma} G^{a \mu v} G^{a \rho \sigma}$ is the topological density operator, such that local $\mathcal{P}$ and $C \mathcal{P}$ invariance of QCD is broken on the scales where correlated state with $\left\langle\theta(\vec{x}, t)_{\text {ind }}\right\rangle \neq 0$ is induced. One should expect a number of $\mathcal{P}$ and $C \mathcal{P}$ violating effects taking place in a relatively large region where $\left\langle\theta(\vec{x}, t)_{\text {ind }}\right\rangle \neq 0$.

The first question to be addressed in the present work is as follows: what is the physics behind of this long range order in the system with a gap $\sim \Lambda_{\mathrm{QCD}}$ ? As it is known, normally, if the system has a gap, conventional correlation functions decay exponentially fast with a typical scale of order of $\Lambda_{\mathrm{QCD}}^{-1}$

ae-mail: arz@phas.ubc.ca 
rather than demonstrate a long range order which is normally associated with a massless particle. The second question to be addressed in the present work can be formulated as follows. Let us assume that the long range order is indeed present in the system. Than, how one can observe it?

One should comment here that the charge separation effect, CME, chiral separation effect and many other related phenomena have been very active area of research in recent years, see review paper [9] with large number of references on the original results. However, the key ingredient of the entire framework, the presence of the long range structure in the system, has not been properly addressed in previous studies ${ }^{1}$, with very few exceptions. The goal of this work is to explain the emergence of this long range order and present few profound observational consequences which might be related to this long range structure. In our presentation we follow the original results on the subject [12-16].

Our presentation is organized as follows. We start in section 2 with definition of the key element of our study-the non-dispersive contact term in topological susceptibility. The unique feature of this contact term is that it does contribute to the $\theta$ dependent portion of the vacuum energy $E_{\mathrm{vac}}(\theta)$. However, this term can not be expressed in terms of any physical propagating degrees of freedom, therefore, it has a non-dispersive nature. Exactly this fundamentally new type of the vacuum energy is highly sensitive to the long distance physics, and will play the key role in our applications considered in section 3 and 4. Specifically, in section 2 we shall argue that the long range sensitivity is related to the non-perturbative dynamics of the topologically nontrivial sectors in gauge theories [12]. In section 3 we apply our findings on long range order to heavy ion collisions [13, 14], while in section 4 we consider some cosmological applications. Specifically, we shall argue $[15,16]$ that the de Sitter phase may dynamically emerge in strongly coupled gauge theories as a result of dynamics of the topologically nontrivial sectors in expanding universe. If this effect is confirmed by future analytical and numerical studies (which are presently underway), it may have profound consequences for our understanding of the inflationary phase in early inverse. It may also shed some light on the dynamics of the dark energy we are witnessing now.

\section{Contact term, and the Long Range Order in QCD}

We introduce the topological susceptibility $\chi$ which plays a crucial role in resolution of the $U(1)_{A}$ problem in QCD [17-19] as follows ${ }^{2}$

$$
\chi(\theta=0)=\left.\frac{\partial^{2} E_{\mathrm{vac}}(\theta)}{\partial \theta^{2}}\right|_{\theta=0}=\lim _{k \rightarrow 0} \int \mathrm{d}^{4} x e^{i k x}\langle T\{q(x), q(0)\}\rangle,
$$

where $\theta$ parameter enters the Lagrangian along with topological density operator $q(x)$ and $E_{\mathrm{vac}}(\theta)$ is the $\theta$ dependent portion of the vacuum energy density in QCD. It is important that the topological susceptibility $\chi$ does not vanish in spite of the fact that $q=\partial_{\mu} K^{\mu}$ is total divergence. This feature is very different from any conventional correlation functions which normally must vanish at zero momentum if the corresponding operator can be represented as total divergence. Furthermore, any physical massive state (meson, or glueball) gives a negative contribution to this diagonal correlation

\footnotetext{
${ }^{1}$ In particular, for the effective Lagrangian approach advocated in the original proposal [8] (as well as in many followup papers) to be justified, the corresponding slow parameter $\theta(\vec{x}, t)_{\text {ind }} \neq 0$ may only fluctuate with frequencies which are much smaller than $\Lambda_{\mathrm{QCD}}$. Otherwise, the effective Lagrangian approach breaks down. The presence of the new scale of the problem can be formally expressed as $\left|\partial_{\mu} \theta_{\text {ind }}\right| \sim R^{-1} \ll \Lambda_{\mathrm{QCD}}$, where parameter $R$ can be identified with the size of the system. The problem on the long range order in this framework can be formulated as follows: how one could understand the emergence of the new scale $\sim R$ in the system with the gap $\sim \Lambda_{\mathrm{QCD}}$ ? The same question in terms of the axial chemical potential defined as $\mu_{5} \equiv \dot{\theta}_{\text {ind }}[9,11]$ can be formulated as follows: why $\mu_{5}$ is coherently correlated on a large scale of order $\sim R$ ?

${ }^{2}$ We use the Euclidean notations where path integral computations are normally performed.
} 
function

$$
\chi_{\text {dispersive }} \sim \lim _{k \rightarrow 0} \int d^{4} x e^{i k x}\langle T\{q(x), q(0)\}\rangle \sim \lim _{k \rightarrow 0} \sum_{n} \frac{\langle 0|q| n\rangle\langle n|q| 0\rangle}{-k^{2}-m_{n}^{2}} \simeq-\sum_{n} \frac{\left|c_{n}\right|^{2}}{m_{n}^{2}} \leq 0,
$$

where $m_{n}$ is the mass of a physical $|n\rangle$ state, $k \rightarrow 0$ is its momentum, and $\langle 0|q| n\rangle=c_{n}$ is its coupling to topological density operator $q(x)$. At the same time the resolution of the $U(1)_{A}$ problem requires a positive sign for the topological susceptibility (1), see the original reference [19] for a thorough discussion,

$$
\chi_{\text {non-dispersive }}=\lim _{k \rightarrow 0} \int \mathrm{d}^{4} x e^{i k x}\langle T\{q(x), q(0)\}\rangle>0 .
$$

Therefore, there must be a contact contribution to $\chi$, which is not related to any propagating physical degrees of freedom, and it must have the "wrong" sign. The "wrong" sign in this paper implies a sign which is opposite to any contributions related to the physical propagating degrees of freedom (2). The vacuum energy $E_{\mathrm{vac}}(\theta)$ which is expressed in terms of $\chi$ according to eq. (1) can not be formulated in terms of any conventional propagating degrees of freedom as it has pure non-dispersive nature according to eqs. $(2,3)$.

In the framework [17] the contact term with "wrong" sign has been simply postulated, while in refs. $[18,19]$ the Veneziano ghost (with a "wrong" kinetic term) had been introduced into the theory to saturate the required property (3). Furthermore, as we discuss below the contact term has the structure $\chi \sim \int d^{4} x \delta^{4}(x)$. The significance of this structure is that the gauge variant correlation function in momentum space

$$
\lim _{k \rightarrow 0} \int d^{4} x e^{i k x}\left\langle K_{\mu}(x), K_{v}(0)\right\rangle \sim \frac{k_{\mu} k_{v}}{k^{4}}
$$

develops a topologically protected "unphysical" pole which does not correspond to any propagating massless degrees of freedom, but nevertheless must be present in the system. Furthermore, the residue of this pole has the "wrong sign", which precisely corresponds to the Veneziano ghost contribution saturating the non-dispersive term in gauge invariant correlation function (3),

$$
\langle q(x) q(0)\rangle \sim\left\langle\partial_{\mu} K^{\mu}(x), \partial_{\nu} K^{v}(0)\right\rangle \sim \delta^{4}(x) .
$$

We should comment here, that the entire framework, including the singular behaviour of $\langle q(x) q(0)\rangle$ with the "wrong sign", has been accepted by the community as a standard resolution of the $U(1)_{A}$ problem which has been well confirmed by numerous lattice simulations in strongly coupled regime, see e.g. recent papers [20-22] and many references therein. Furthermore, it has been argued long ago in [23] that the gauge theories may exhibit the "secret long range forces" (which is, in fact, the title of ref.[23]) expressed in terms of the topologically protected massless pole in correlation function (4).

While the the presence of the contact term (3) in the topological susceptibility in QCD is well established fact, its nature and origin still remain a mystery even today. Indeed, answers on the questions on nature of the vacuum configurations saturating the topological susceptibility (3) are still unknown in strongly coupled QCD as the corresponding answers are hard to extract from the numerical QCD lattice simulations.

Fortunately, all relevant questions can be addressed in a weakly coupled gauge theory, the socalled "deformed QCD" [24]. This model is a weakly coupled gauge theory due to some special deformations as constructed in [24]. However, this model preserves all crucial ingredients of the original theory, such as confinement, the presence of the topological sectors, the $\theta$ dependence, etc. which allow to perform the analytical computations in theoretically controllable way. It allows us 
to answer the crucial questions about the vacuum configurations saturating the contact term in this model. Furthermore, it allows us to understand and illuminate the infra-red (IR) nature of the contact term, and demonstrate its sensitivity to the large distance physics in spite of the fact that the system is gapped as a result of confinement in this model.

To be more specific, the topological susceptibility and the vacuum energy in the "deformed QCD" model can be explicitly calculated, and it is saturated by fractionally charged weakly interacting monopoles when semiclassical computations are under complete theoretical control. The result for $|\theta| \leq \pi$ is $[12,25]$ :

$$
\chi_{Y M}=\frac{\zeta}{N L} \int d^{3} x[\delta(\mathbf{x})], \quad E_{\mathrm{vac}}(\theta)=-\frac{N \zeta}{L} \cos \frac{\theta}{N},\langle q(x)\rangle \equiv-i \frac{\partial E_{\mathrm{vac}}(\theta)}{\partial \theta}=-i \frac{\xi}{L} \sin \frac{\theta}{N} .
$$

In this formula $N$ stands for number of colours, $\zeta$ is the monopole fugacity of the system, which is assumed to be parametrically small for semiclassical approximation to be justified, and parameter $L$ is the size of compact dimension in the Euclidean time direction.

The topological susceptibility has the required "wrong sign" as this contribution is not related to any physical propagating degrees of freedom, and it has a $\delta(\mathbf{x})$ function structure which implies the presence of the pole (4). However, there are no any physical massless states in the system as it is gapped. This contact term (6) is described in terms of the tunnelling events between different (but physically equivalent) topological sectors $|k\rangle$ which always present in gauge systems. The monopoles in this framework are not real particles, they are pseudo-particles which live in Euclidean space-time and describe the physical tunnelling processes between different topological sectors $|k\rangle$ and $|k+1\rangle$. The vacuum energy of the system (6) should be interpreted as number of tunnelling events per unit time $L$ per unit volume $V$ as explained in [12,25]. Precisely this interpretation reveals the non-dispersive nature of this vacuum energy which can not be attributed to any physical propagating degrees of freedom. It is quite obvious that the nature of this energy is very different from conventional vacuum energy formulated in terms of any dynamical propagating field.

Furthermore, the $\delta(\mathbf{x})$ function in (6) should be understood as total divergence related to the IR physics, rather than to ultraviolet (UV) behaviour as explained in [25]

$$
\chi_{Y M} \sim \int \delta(\mathbf{x}) d^{3} x=\int d^{3} x \partial_{\mu}\left(\frac{x^{\mu}}{4 \pi x^{3}}\right)=\oint_{S_{2}} \mathrm{~d} \Sigma_{\mu}\left(\frac{x^{\mu}}{4 \pi x^{3}}\right) .
$$

In other words, the non-dispersive contact term with the "wrong" sign (6) is highly sensitive to the boundary conditions and behaviour of the system at arbitrarily large distances. Therefore, it is natural to expect that a variation of the boundary conditions or placement this system into a time dependent background would change the topological susceptibility (6) and associated with it the vacuum energy $E_{\text {vac }}$ despite of the fact that the system has a gap.

Our final remark in this section is as follows. We observe the long range sensitivity of the system in terms of topologically protected pole (4), or what is the same, in terms of the surface integral (7) despite the presence of the gap in the system which itself reflects the property of the confinement in this model. One can describe the same physics, including the behaviour in the IR region (4) using the auxiliary topological non-propagating fields as developed in ref. [12]. In fact the corresponding construction [12] in "deformed QCD" model sheds some lights on the nature of the Veneziano ghost $[18,19]$ which was introduced ad hoc in QCD long ago with the only purpose to saturate the correlation function (4) with a "wrong sign". One can explicitly see in this "deformed QCD" model that auxiliary topological non-propagating fields can be explicitly identified with the Veneziano ghost as these massless auxiliary fields exactly saturate the topological susceptibility without violation unitarity, casualty or any other fundamental properties of quantum field theory. This representation further 
illuminates the IR nature of the contact term as the auxiliary non-propagating topological fields are massless and indeed develop a pole at $k=0$ in eq. (4). Furthermore, based on this construction, one can argue that QCD belongs to a topologically ordered phase, similar to many known examples in condensed matter physics, see [12] for the details.

\section{$3 \mathcal{P}$-odd fluctuations and Long Range Order in Heavy lon Collisions}

In previous section we interpreted the vacuum configurations which saturate the topological susceptibility (6) and associated with it the vacuum energy $E_{\mathrm{vac}}$ as tunnelling processes which are happening all the time in Minkowski vacuum with no interruptions. These tunnelling processes in Minkowski vacuum (when no any external sources are present in the system) do not lead to any emission or absorption of real particles, similar to the persistent tunnelling events in Bloch's case in condensed matter physics. These tunnelling events simply select an appropriate ground state of the system which is a specific superposition of $|k\rangle$ states. While each gauge configuration has definite sign of the topological charge density, the opposite sign alternate. This delicate cancellation between the opposite sign topological configurations leads to the known result corresponding to $\langle q(x)\rangle=0$ in the physical unperturbed vacuum at $\theta=0$.

However, when some external sources are present in the system a delicate cancellation between the opposite sign topological configurations may lead, in general, to a local minimum with nontrivial values for $\langle q(x)\rangle \neq 0$ in the region $R \sim 10 \mathrm{fm}$ where external impact is felt, i.e. in the entire volume of collision region. A non-vanishing expectation value for the topological density (which is $\mathcal{P}$ and $C \mathcal{P}$ odd operator) in large region of space-time can be interpreted, according to eq. (6) as a formation of the $\left|\theta_{\text {ind }}\right\rangle$ local vacuum state in terminology $[8,9]$. In fact, one can explicitly show in simplified "deformed QCD" model that such local minima with nontrivial values of $\langle q(x)\rangle \neq 0$ indeed exist in the system, see [26] for the details.

This is precisely the basic idea advocated in refs. $[13,14]$ that the long range structure expressed by (4),(6) may lead to local violation of the $\mathcal{P}$ and $C \mathcal{P}$ invariance in the same region $R$ where $\langle q(x)\rangle \neq 0$ is correlated. This mechanism is very different from all conventional models (such as sphaleron transitions) which are typically characterized by a finite correlation length $\sim \Lambda_{\mathrm{QCD}}^{-1}$. Those models normally predict a parametrically small magnitude $\sim \exp \left(-\Lambda_{\mathrm{QCD}} R\right)$ for coherent effects considered below, while our mechanism predicts the intensity to be proportional $\sim R^{-1}$ where $R \sim 10 \mathrm{fm}$ is a size of the system.

There is a number of generic consequences of this framework which have been discussed in details in refs. $[13,14]$. We list them below:

a) The thermal spectrum in $e^{+} e^{-}, p p$ and $p \bar{p}$ high energy collisions emerges in spite of the fact that the statistical thermalization could never be reached in those systems. An approximate universality of the temperature with no dependence on energy of colliding particles nor their nature (including $e^{+} e^{-}, p p$ and $p \bar{p}$ collisions) is due to the fact that the emission occurs from the distorted QCD vacuum state represented by the long range vacuum configurations, discussed above, rather than from the colliding particles themselves. In heavy ion collisions there is, of course, a conventional thermal component, in addition to the emissions due to the tunnelling transitions between topological $|k\rangle$ sectors.

b) The intensities of the correlations ${ }^{3}$ due to the local $\mathcal{P}$ violation should demonstrate the universal behaviour similar to the "universal apparent thermalization" discussed in item a) above as the

\footnotetext{
${ }^{3}$ The basic observables which measure corresponding asymmetries on event by event basis have been originally suggested in [1] and extensively studied in [2-7]. We shall not discuss the corresponding definitions of the observables in the present work by referring to the original papers.
} 

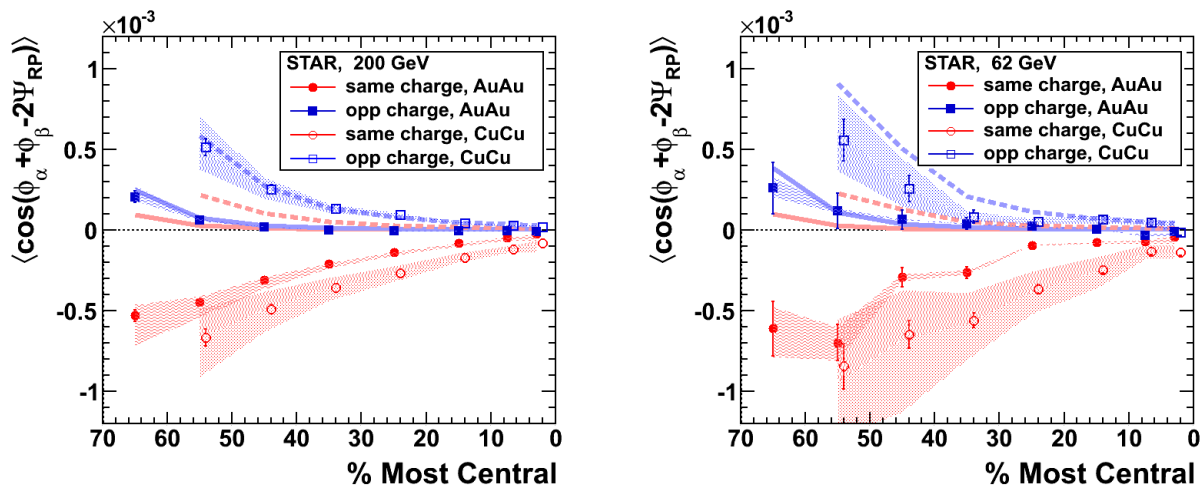

Figure 1. Data for $\sqrt{s_{N N}}=200 \mathrm{GeV}$ and $\sqrt{s_{N N}}=62 \mathrm{GeV}$ for $\mathrm{Au}+\mathrm{Au}$ and $\mathrm{Cu}+\mathrm{Cu}$ collisions (adapted from [2]). The plots demonstrate the universality in behaviour, see text for the details.

source for the both effects is the same and related to the emission of the particles due to the distorted tunnelling transitions between topological $|k\rangle$ sectors. In particular, the effect should not depend on energy of colliding ions. Such independence on energy is indeed supported by observations where correlations measured in $\mathrm{Au}+\mathrm{Au}$ and $\mathrm{Cu}+\mathrm{Cu}$ collisions at $\sqrt{s_{N N}}=62 \mathrm{GeV}$ and $\sqrt{s_{N N}}=200 \mathrm{GeV}$ are almost identical and independent on energy, see Fig. 1. We expect the same tendency to continue for the LHC energies. The recent results from ALICE Collaboration [4-7] indeed confirm this prediction.

c) For a system of size $R \gg \Lambda_{\mathrm{QCD}}^{-1}$ the observable $\mathcal{P}$ odd effect due to the collisions are expected to be proportional to $R^{-1}$, see [13] for the details. The $R^{-1}$ scaling essentially describes the deviation of the system from the ground state in the region $R$ as a result of collision. As one can see from Fig. 1 some suppression of the measured correlations with increasing the size of the system indeed has been observed. We would like to interpret this suppression as a manifestation of the $R^{-1}$ scaling. Indeed, the effect for $\mathrm{Au}+\mathrm{Au}$ collisions with $A \simeq 197$ is obviously suppressed in comparison with $\mathrm{Cu}+\mathrm{Cu}$ collisions with $A \simeq 64$. There are many other effects which influence this ratio (both systems are obviously not very large when derivative expansion used in [13] is justified). However, the effect goes in the right direction (the effect is stronger for a smaller size system $\mathrm{Cu}+\mathrm{Cu}$ than for a larger $\mathrm{Au}+\mathrm{Au}$ system).

Order of magnitude estimate for the observed asymmetries can be presented as follows

$$
\text { [asymmetries on Figs. 1] } \sim \frac{e}{N_{c}^{2}} \cdot \frac{f}{R \Lambda_{\mathrm{QCD}}} \sim 10^{-3},
$$

where $e$ is the electric charge $e \sim \sqrt{\alpha} \sim 10^{-1}$, while $\sim 1 / N_{c}^{2} \sim 10^{-1}$ is a typical suppression factor which accomponies any phenomena related to the $\theta$ dependence, see [13] with a more accurate esti- 
mate for this suppression factor. Finally, factor $\left(R \Lambda_{\mathrm{QCD}}\right)^{-1} \sim 10^{-1}$ is the key element of the entire framework: all observed asymmetries would be much smaller $\sim \exp \left(-R \Lambda_{\mathrm{QCD}}\right)$ if the long range order (leading to the algebraic $\sim R^{-1}$ decay) is not present in the system. Finally, parameter $f \sim 1$ depends on a number of different time scales relevant for the problem, see [13] for the details.

The estimate (8) is the direct consequence of the long range order in QCD formally expressed by eq. (4) which explicitly demonstrates the presence of topologically protected pole at $k=0$. As we discussed in section 2 the corresponding IR sensitivity is a result of dynamics of the topological sectors $|k\rangle$ in QCD. Our application to heavy ion collisions is based on an idea that the dynamics of the vacuum topological sectors is slightly (but coherently) modified on the scale of order $R \sim(10 \mathrm{fm})$ as a result of a collision (which itself is treated as a time-dependent external impact on the system size $R$ ). We have also argued that the corrections to the vacuum energy density due to the long range order may receive some power-like corrections of order $1 / R$ in contrast with a naive expectation $\exp (-R)$ related to the conventional fluctuations with finite mass $\sim \Lambda_{\mathrm{QCD}}$. As a result of such $1 / R$ scaling we estimate that the intensity for the coherent effects to the local violation of the $\mathcal{P}$ and $C \mathcal{P}$ invariance could be quite large (8).

To conclude this section: the basic, model-independent consequences of the entire framework listed above are consistent with all available data. Furthermore, the qualitative predictions of this framework (e.g. on energy independence of the observables at the LHC energies) have been confirmed by the ALICE Collaboration [4-7]. Order of magnitude estimate (8) is also consistent with the results from RHIC and the LHC.

\section{Topological Long Range Order and the de Sitter Accelerating Universe}

In this section we consider another application related to the long range order in QCD formally expressed by eq. (4) demonstrating the presence of topologically protected pole at $k=0$. To be more concrete, we want to consider external time-dependent background characterized by the Hubble constant $H \sim 10^{-33} \mathrm{eV}$ which is drastically different from the scale $R^{-1} \sim(10 \mathrm{fm})^{-1}$ we dealt with in previous section 3. We anticipate some slight modification of the QCD vacuum topological sectors $|k\rangle$ as a result of slow expansion with rate $H$. Our goal here is to study the tiny variation of the ground state energy which may have profound cosmological consequences.

The logic of our approach remains the same as in section 3, with the only difference is that instead of considering time-dependent external impact on the system with size $R$ as a result of ion collisions we are now considering a system with a size of visible Universe $H^{-1}$ in a time dependent background characterized by the Hubble constant $H$. As in our previous analysis in section 3, we try to understand the qualitative behaviour of the system using a simplified "deformed QCD" model, which is weakly coupled gauge theory but preserves all relevant elements, such as confinement, topological sectors, $\theta$ dependence, etc, of strongly coupled QCD.

To be more specific, we address the following very hard question: How does the non-dispersive vacuum energy (6), and the corresponding contact term in $\chi$ vary when the system couples to the gravity? We can rephrase and simplify the same question as follows: how does the rate of tunnelling processes change when the system is considered in a time-dependent background? In principle, the strategy to carry out the corresponding computations is as follows. First, find the classical solution in a nontrivial background which generalize the fractionally charged monopoles saturating the contact term (6) along the line of ref.[25]. Next, one should compute the corresponding path integral measure in semiclassical approximation which must depend now on the parameters of a background such as the Hubble constant $H$. The corresponding corrections (due to the background) in the coefficients of the effective action can be translated to the corresponding corrections in vacuum energy, which represents the desired result. 
Unfortunately, a complete resolution of this program is not even feasible. However, there are few general arguments which may provide us with some hints on possible dependence of the nondispersive energy (6) from a background which will be parameterized in what follows by the Hubble parameter $H$. In general, one should expect that for sufficiently weak background (which we always assume to be the case) the correction to all observables, including the vacuum energy, can be represented as the power corrections of $H$, i.e.

$$
E_{\mathrm{vac}}(H)=\sum_{p} c_{p} H^{p}, \quad c_{0}=-\frac{N \zeta}{L},
$$

where $c_{0}$ is given by (6) computed in flat background.

There is a number of generic arguments which suggest that $p$ in eq. (9) can only be even, i.e. $p=0,2,4 \ldots$ The generic arguments, as usual, may have some loopholes.... We refer to ref.[15] with detail discussions of pros and cons of these arguments. The only comment we want to make here is that an assumption of locality supporting $p=0,2,4 \ldots$ might not be so harmless for non-abelian gauge theories such as QCD, in contrast with a simple example of a massive scalar field theory. Indeed, while QCD has a gap in gauge invariant sectors, it nevertheless demonstrates a high IR sensitivity in gauge variant sectors in terms of a topologically protected massless pole (4). This pole is not screened by the confinement mechanism, and in fact, is the manifestation of the inherently non-local large gauge transformation operator ${ }^{4}$ which itself is the key element in the mechanism of generating the nondispersive vacuum energy $E_{\text {vac }}$. Furthermore, with few additional simplifications one can explicitly see how the linear $\sim H$ correction may indeed emerge in the "deformed QCD" model, see Appendix in ref. [15] with some technical details. Finally, the linear $\sim H$ correction has been computed using the Monte Carlo lattice numerical simulations in strongly coupled regime [27], see Fig.2 adapted from that paper.

Why is the linear correction $\sim H$ to the vacuum energy $E_{\mathrm{vac}}(H)$ so crucial for our discussions? A simple answer is that precisely such kind of corrections will drag the Universe into de Sitter state as we shall explain below.

\subsection{The de Sitter Accelerating Universe. Dark Energy.}

In what follows we assume that the linear term $\sim H$ is indeed generated in the expression for the vacuum energy in strongly coupled QCD, such that $E_{\mathrm{FLRW}}(H)$ in context of the Friedmann-LemaitreRobertson-Walker (FLRW) Universe takes the following form

$$
E_{\mathrm{FLRW}}(H)=\left[\Lambda_{\mathrm{QCD}}^{4}+H \Lambda_{\mathrm{QCD}}^{3}+O\left(H^{2}\right)\right],
$$

where the first term $\Lambda_{\mathrm{QCD}}^{4}$ is well known and well studied expression for the vacuum energy computed in the flat background, i.e. $E_{\mathrm{Mink}}=\Lambda_{\mathrm{OCD}}^{4}$. The presence of a linear term $\sim H$ in this expression is analogous to $1 / R$ factor in eq. (8), and they are both generated due to the long range order present in the system, as discussed in section 2 .

As we already explained previously the energy (10) is saturated by tunnelling transitions between physically identical but topologically distinct topological sectors $|k\rangle$. The physics of tunnelling processes and the corresponding generated energy (10) can not be described in terms of any local dynamical field $\Phi(x)$, as the tunnelling between topologically distinct sectors is fundamentally non-local

\footnotetext{
${ }^{4}$ The large gauge transformation operators is defined as follows $\mathcal{T}|k\rangle=|k+1\rangle$. It commutes with the Hamiltonian, $[H, \mathcal{T}]=$ 0 . The non-dispersive vacuum energy (6) is non-perturbative in nature as it is generated as a result of tunnelling events between $|k\rangle$ and $|k+1\rangle$ topological sectors connected by non-local operator $\mathcal{T}$.
} 

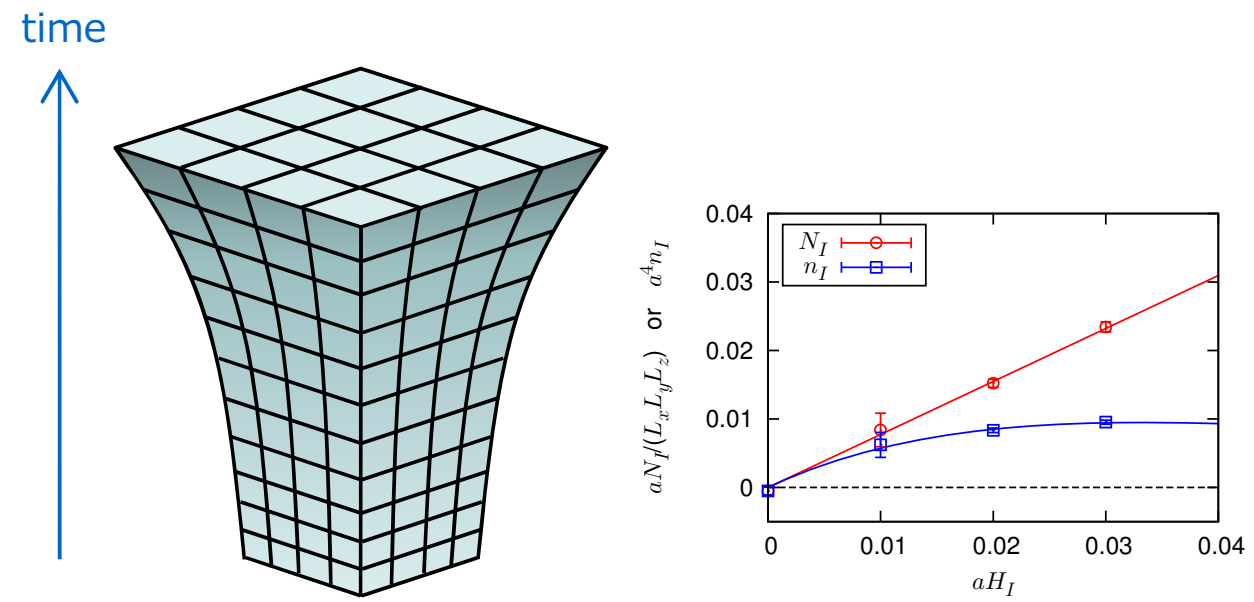

Figure 2. Expanding lattice (on the left) and $H$ dependence of the produced particles per unit volume per unit time as a result of this expansion (on the right). The rate of particle production is shown as a function of the expansion rate $H$ in units of the lattice size $a$. It clearly shows the linear dependence of an observable on $H$, in complete agreement with our eq. (10). The plots adapted from [27].

phenomenon as it is described in terms of non-local operator $\mathcal{T}$, see footnote 4 . Therefore, the energy (10) can not be expressed in terms of any local operators such as curvature, which would be a conventional structure to emerge when physical propagating degrees of freedom are integrated out in the background of the gravitational field. This feature is similar to the well known property of a topologically ordered phase in condensed matter physics wherein an expectation value of a local operator does not characterize the system. Instead, a system should be described in terms of some non-local variables such as holonomy, see $[15,16]$ for the details.

Our next assumption, in addition to (10), can be formulated as follows. We adopt the paradigm that the relevant definition of the energy which enters the Einstein equations in an expanding background (characterized by the parameter $H$ ) is the difference $\Delta E(H) \equiv\left[E(H)-E_{\text {Mink }}\right]$, similar to the definition of the Casimir energy. This element in our analysis is not very new, and in fact in the present context such a definition for the vacuum energy was advocated for the first time in 1967 by Zeldovich [28], see [29] for review and references on this matter.

With these assumptions just formulated, the Universe has a period of the de Sitter phase expansion characterized by the exponential growth of the scale factor a $(t) \sim \exp \left(H_{0} t\right)$. Indeed, the Friedman equation assumes the following form

$$
H^{2}=\frac{8 \pi G}{3}\left(\rho_{\mathrm{DE}}+\rho_{R}+\rho_{M}\right)=\frac{8 \pi G}{3}\left(H \Lambda_{\mathrm{QCD}}^{3}+\rho_{R}+\rho_{M}\right),
$$

where we identify $\rho_{\mathrm{DE}}$ with $\Delta E(H)=\left[E(H)-E_{\mathrm{Mink}}\right]=\left[E(H)-\Lambda_{\mathrm{QCD}}^{4}\right]$ from (10). Furthermore, the corresponding energy density according to eq. (10) is given by $\rho_{\mathrm{DE}}=H \Lambda_{\mathrm{QCD}}^{3}$. Two other components: $\rho_{R}$ and $\rho_{M}$ are the radiation and matter (including dark matter) components of the total energy of the system. In (11) we neglected higher order corrections $O\left(\Lambda_{\mathrm{QCD}}^{2} H^{2}\right)$ in the expansion (10) as $H \ll \Lambda_{\mathrm{QCD}}$. The radiation and matter components in eq. (11) scales as $\rho_{R} \sim \mathrm{a}^{-4}$ and $\rho_{M} \sim \mathrm{a}^{-3}$ correspondingly, such that $\rho_{\mathrm{DE}}$ starts to dominate the universe at some point when $H$ approaches the 
constant value $H_{0}$ estimated as follows, which is amazingly close to the observed value

$$
H_{0} \simeq \frac{8 \pi G \Lambda_{\mathrm{QCD}}^{3}}{3} \simeq \frac{\Lambda_{\mathrm{QCD}}^{3}}{3 M_{\mathrm{PL}}^{2}}, \quad H_{0} \sim 10^{-33} \mathrm{eV}, \quad \rho_{\mathrm{DE}} \sim H_{0} \Lambda_{\mathrm{QCD}}^{3} \sim\left[\left(10^{-2}-10^{-3}\right) \mathrm{eV}\right]^{4}
$$

The constant $H_{0}$, which is unambiguously determined by the strongly coupled dimensional parameter $\Lambda_{\mathrm{QCD}}$ corresponds to the de Sitter behaviour a $(t) \sim \exp \left(H_{0} t\right)$ as claimed.

Few comments are in order. First, eq. (12) is obviously an oversimplified estimate as we do not take into account a number of important elements. In particular, we completely ignore the presence of the light quarks in the system which would lead to factor $m_{q} \sim 5 \mathrm{MeV}$ instead of $\Lambda_{\mathrm{QCD}} \sim 100$ $\mathrm{MeV}$. Furthermore, the computation of the numerical coefficient in front of $\sim H$ in eq. (10), which eventually enters the Friedman equation (11) is a very hard problem. It is obviously the prerogative of numerical Monte Carlo lattice simulations similar to computations performed recently in [27], rather than a subject of analytical calculations. We think it is next to impossible to perform analytical computations of this coefficient even in simplified version of QCD, the "deformed QCD" as explained at the very beginning of this section. Still, an order of magnitude estimate (12) looks very promising and very encouraging, and obviously warrants a further investigation along this line of thinking.

Our next comment is as follows. We advocate a new paradigm which is based on a fundamentally novel view on the nature and origin of the dark energy (DE) corresponding to the de Sitter behaviour with constant $H_{0}$ and constant dark energy density $\sim H_{0} \Lambda_{\mathrm{QCD}}^{3}$ which effectively imitates the cosmological constant on the time scale when $H_{0}$ is almost constant (12). This paradigm is drastically different from the conventional viewpoint that DE field is a dynamical local field ${ }^{5}$. In our new framework the DE is a genuine quantum effect, and the DE effective field is an auxiliary topological field ${ }^{6}$. The corresponding physics is fundamentally indescribable in terms of any local propagating fields, such as a local scalar field $\Phi(x)$ which is normally used by cosmology- practitioners to describe the DE. One should note that a similar auxiliary field, for example, is known to emerge in the description of the topologically ordered condensed matter (CM) systems realized in nature.

\subsection{The de Sitter Accelerating Universe. Inflation.}

It is well known that the deep issue inflation addresses (among many other things) is the origin of the large-scale homogeneity of the observable universe[30-32]. The crucial element of this idea is to have a period of evolution of the universe which can be well approximated by the de Sitter behaviour. In this case the scale parameter $\mathrm{a}(t)$ and the equation of state takes the following approximate form,

$$
\mathrm{a}(t) \sim \exp (H t), \quad \rho \approx-p, \quad H \equiv \frac{\dot{\mathrm{a}}}{\mathrm{a}} \approx \mathrm{constant}
$$

\footnotetext{
${ }^{5}$ The model (11) has been successfully confronted with observations and it has been claimed that this proposal is consistent with all presently available data, see references on original works with some comments in [15]. An interesting unique prediction of this model, yet to be tested, is that the Universe must demonstrate some sort of the $\mathcal{P}$ odd phenomena on the largest possible scales order of size of the visible Universe $\sim H^{-1}$, similar to our discussions on the local $\mathcal{P}$ violation in heavy ion collisions in section 3. In fact, there are many claims (from observations) apparently pointing towards a large scale violation of statistical isotropy in our Universe, such as "cosmic axis of evil", etc. However, I think it is too premature to make any solid statements on this matter.

${ }^{6}$ This field does not propagate, does not have a canonical kinetic term, as the sole role of the auxiliary field is to effectively describe the dynamics of the topological sectors of a gauge theory saturating the correlation functions (3), (4), (5) in a timedependent background. These auxiliary fields are not mandatory fields, but instead play a supplementary role to simplify the analysis of the dynamics of the multiple tunnelling transitions between the distinct topological sectors in strongly coupled QCD. A similar well-known example where the emergent (auxiliary) fields are introduced into the system is the quantum Hall effect. In this case the dynamics of the auxiliary fields are controlled by the Chern-Simons effective Lagrangian.
} 
It is commonly accepted that such equation of state can be achieved in quantum field theory by assuming the existence of a local scalar matter field $\Phi(x)$, the inflaton, with a non-vanishing potential energy density $V[\Phi(x)]$. The shape of this potential energy can be adjusted in a such a way that the contribution to energy density $\rho$ and pressure $p$ is in agreement with the above equation of state.

We advocate a fundamentally different view on the nature and origin of the inflaton field $[15,16]$. Our proposal is based on assumption that the inflaton is an auxiliary field of a strongly coupled gauge theory, very much the same as we discussed in previous section with application to the dark energy (DE), see footnote 6 with some comments. However, the scale of inflation and scale of the present DE density (12) are drastically different. Therefore, we assume there existence of a scaled up version of QCD (which is coined in ref. [15] as $\overline{\mathrm{QCD}}$ ) determined by high inflationary scale $\Lambda_{\overline{\mathrm{QCD}}}<M_{P L}$, to be determined from observations. A similar construction (though in a different context) had been suggested long ago for a different purpose and is known as technicolor.

Therefore, one can repeat the arguments from the previous section 4.1 to conclude that the Friedman equation has a non-trivial solution $H_{0} \simeq$ constant,

$$
H^{2}=\frac{8 \pi G}{3}\left(\rho_{\mathrm{Inf}}+\rho_{R}\right)=\frac{8 \pi G}{3}\left(H \Lambda_{\overline{\mathrm{QCD}}}^{3}+\rho_{R}\right), \Rightarrow H_{0} \simeq \frac{8 \pi G \Lambda_{\overline{\mathrm{QCD}}}^{3}}{3}, \quad \rho_{\mathrm{Inf}} \simeq \frac{8 \pi G \Lambda_{\overline{\mathrm{QCD}}}^{6}}{3} .
$$

This solution $H_{0} \simeq$ constant is identified with inflationary de Sitter behaviour (13). In our new framework the inflation is a genuine quantum effect while the inflaton is an auxiliary topological field, see footnote 6 with few comments on the nature of this field.

The inflationary regime described by eqs. (13) and (14) would be the final destination of our Universe if the interaction of the $\overline{\text { QCD }}$ fields with the Standard Model (SM) particles were always switched off. When the coupling is switched back on, the end of inflation is triggered precisely by this interaction which itself is unambiguously fixed by the triangle anomaly as we discuss below. This is so-called the reheating period. The only information which is needed for the future discussions is that the auxiliary topological field $b(x)$, generating the corresponding inflaton-related energy (14) couples to the SM particles precisely in the same way as the $\theta$ parameter couples to the gauge fields, see [15] for the details. In other words, the coupling is

$$
\mathcal{L}_{b \gamma \gamma}(x)=\frac{\alpha\left(H_{0}\right)}{8 \pi} N Q^{2}[\theta-b(x)] \cdot F_{\mu \nu} \tilde{F}^{\mu v}(x),
$$

where $\alpha\left(H_{0}\right)$ is the fine-structure constant measured during the period of inflation, $Q$ is the electric charge of a $\overline{\mathrm{QCD}}$ quark, $N$ is the number of colours of the strongly coupled $\overline{\mathrm{QCD}}$, and $F_{\mu \nu}$ is the usual electromagnetic field strength. The coupling of the $b(x)$ with other $E \& W$ gauge bosons can be unambiguously reconstructed as explained in [15], but we keep a single $E \& M$ field $F_{\mu \nu}$ to simplify the notations and emphasize on the crucial elements of this coupling which is unambiguously fixed by quantum anomaly. We take $\theta=0$ in eq. (15) as we do not intend to discuss in this work an interesting, but different, subject related to the dynamics of the physical axion field.

As we explained above the $b(x)$-field (which is not dynamical, but rather, an emergent auxiliary field, see footnote 6 with few comments) should be treated as a coherent field representing the rate of tunnelling events in the system. It varies and fluctuates as a consequence of expansion, rather than a result of the presence of a kinetic term. As a result of these fluctuations in time dependent background $b(x)$ field radiates real physical particles in expanding universe. This radiation occurs in spite of the fact that $b(x)$ itself is not a dynamical field. This is precisely the way how the inflaton energy (14) can be transferred to the SM particles. Eventually this process of the energy transfer is responsible for the termination of the inflationary epoch.

How long does it take for the energy (14) to be transferred to SM particles? The corresponding rate of transfer is determined by the coupling (15). We estimate the production of real particles in 
expanding background parameterized by the Hubble constant $H_{0}$ as follows

$$
\frac{d P}{d V d t} \sim \alpha^{2}\left(H_{0}\right) H_{0} \Lambda_{\overline{\mathrm{QCD}}}^{3}
$$

The particle production in our system is a result of multiple tunnelling events between the topological sectors $|k\rangle$ in the background of the gravitational field. It should be contrasted with conventional computations during the reheating epoch when the radiation of SM particles occur as a result of dynamics of real propagating inflaton field. As we emphasized previously, the inflaton in our framework is not a propagating degree of freedom, but rather is an auxiliary, non-propagating topological field, see footnote 6 with few comments.

The crucial element in formula (16) is the linear dependence on $H$ which is precisely the same effect as we discussed after eq. (10). The difference between (10) and (16) is that eq. (10) describes the real part of energy momentum tensor, while formula (16) represents the imaginary (absorptive) portion of the energy momentum tensor describing the production of real particles. However, the analyticity suggests that if such term is present in (10) it must be also present in (16), and vice versa. The lattice numerical Monte Carlo simulations also confirm the linear dependence on $H$ for particle production in strongly coupled QCD in the de Sitter background [27]. This computation is consistent with our formula (16) and, therefore, it strongly supports our basic assumption on presence of the linear correction $\sim H$ to the vacuum energy (10).

At the moment $\tau_{\text {Inf }}$ the inflation comes to the end as the dominant portion of the energy is transferred to the light SM particles. Based on particle production rate (16) one could estimate the number of $e$-folds in this mechanism when this moment arrives $[15,16]$,

$$
\tau_{\text {Inf }}^{-1} \sim H_{0} \alpha^{2}\left(H_{0}\right), \quad \Longrightarrow \quad N_{\text {Inf }} \sim \frac{1}{\alpha^{2}\left(H_{0}\right)}
$$

which leads to an estimate $N_{\text {Inf }} \simeq 100$ for $\alpha\left(H_{0}\right) \sim 0.1$, see [16] with more accurate numerical estimate. The key element of this $\overline{\mathrm{QCD}}$ inflationary scenario is that the number of $e$-folds $N_{\text {Inf }}$ in this framework is determined by the gauge coupling constant $\alpha\left(H_{0}\right)$ rather than by dynamics of an ad hoc inflaton $\Phi(x)$ field governed by an ad hoc inflationary potential $V[\Phi(x)]$.

\section{Conclusion}

In the present work we advocate an idea that QCD has some "hidden" long range order which was suspected long ago [23]. It may have profound consequences for our understanding of gauge theories if this long range order will be confirmed by future numerical and analytical computations. In particular, we have argued that the observation of the local violation of $\mathcal{P}$ invariance in heavy ion collisions as discussed in section 3 might be a simple consequence of this long range order. We argue that a sufficiently high intensity of the effect (8) is a result of a coherent modification of the ground state on the scale of nuclei with size $R \sim 10 \mathrm{fm}$, rather than a result of very strong $\mathcal{P}$ odd fluctuation with typical QCD size of $1 \mathrm{fm}$.

Our applications to cosmology considered in section 4.1 may have some profound consequences for our understanding of the Universe we live in. In particular, we argued that the de Sitter behaviour might be dynamically generated as a result of the long range order advocated in this work. In this framework, the $D E$ field is not fundamental local field. Instead, the $D E$ field is an auxiliary topological field which effectively describes the dynamics of topological sectors in QCD when it is considered in the expanding universe. The corresponding energy in this framework has fundamentally different nature than conventional energy when a theory is formulated in terms of a local dynamical field $\Phi(x)$, for example in the Higgs model. In particular, it can not be expressed in terms of any propagating 
physical degrees of freedom as the corresponding energy has a non-dispersive nature. It also can not be expressed in terms of any local variables, as this phenomenon is inherently non-local. The same arguments also apply to the inflaton, see section 4.2.

We conclude this work (mainly devoted to the long range order in QCD and some profound cosmological consequences of this long range order) with the following comment about a different field of physics with drastically different scales. Namely, as we discussed at length in this paper, the heart of the proposal is a fundamentally new type of energy (6), (10) which is not related to any propagating degrees of freedom. Rather, this novel (non-dispersive) contribution to the energy has genuine quantum nature. The effect is formulated in terms of the tunnelling processes between topologically different but physically identical states. This novel type of energy, in fact, has been well studied in the QCD lattice simulations in the flat background, see [15] for references on the original works. Our comment relevant for the present study is that this fundamentally new type of energy can be, in principle, studied in a tabletop experiment by measuring some specific corrections to the Casimir vacuum energy in the Maxwell theory as suggested in [33-35]. This fundamentally new contribution to the Casimir pressure emerges as a result of tunnelling processes, rather than due to the conventional fluctuations of the propagating photons with two physical polarizations. This effect does not occur for the scalar field theory, in contrast with conventional Casimir effect which is operational for both: scalar as well as for Maxwell fields. The extra energy computed in [33-35] is the direct analog of the non-dispersive contribution to the energy (6), (10) which is the key player of the present work. In fact, an extra contribution to the Casimir pressure emerges in this system as a result of nontrivial holonomy which can be enforced by the nontrivial boundary conditions imposed in ref [33-35].

\section{Acknowledgements}

I am thankful to Larry McLerran, Slava Mukhanov, Nemanja Kaloper, and other participants of the International Conference on New Frontiers in Physics (ICNFP), Crete, August 2014, where this work was presented. This research was supported in part by the Natural Sciences and Engineering Research Council of Canada.

\section{References}

[1] S. A. Voloshin, Phys. Rev. C 70, 057901 (2004) [arXiv:hep-ph/0406311].

[2] B. I. Abelev et al. [STAR Collaboration], Phys. Rev. C 81, 054908 (2010) [arXiv:0909.1717 [nucl-ex]].

[3] G. Wang [STAR Collaboration], proceedings for Quark Matter 2012, arXiv:1210.5498 [nucl-ex].

[4] I. Selyuzhenkov [ALICE Collaboration], PoS WPCF 2011, 044 (2011) [arXiv:1203.5230 [nuclex]].

[5] B. Abelev et al. [ALICE Collaboration], Phys. Rev. Lett. 110, 012301 (2013) arXiv:1207.0900 [nucl-ex].

[6] S. A. Voloshin [ALICE Collaboration], Proceedings of the Quark Matter 2012 Conference, Washington D.C., August 2012, arXiv:1211.5680 [nucl-ex].

[7] I. Selyuzhenkov, FAIRNESS 2012 workshop, 3-8 September 2012, Hersonissos, Greece, arXiv:1212.5489 [nucl-ex].

[8] D. Kharzeev and A. Zhitnitsky, Nucl. Phys. A 797, 67 (2007) [arXiv:0706.1026 [hep-ph]].

[9] D. E. Kharzeev, Prog. Part. Nucl. Phys. 75, 133 (2014) [arXiv:1312.3348 [hep-ph]].

[10] D. E. Kharzeev, L. D. McLerran and H. J. Warringa, Nucl. Phys. A 803, 227 (2008) [arXiv:0711.0950 [hep-ph]]. 
[11] K. Fukushima, D. E. Kharzeev and H. J. Warringa, Phys. Rev. D 78, 074033 (2008) [arXiv:0808.3382 [hep-ph]].

[12] A. R. Zhitnitsky, Annals Phys. 336, 462 (2013) [arXiv:1301.7072 [hep-ph]].

[13] A. R. Zhitnitsky, Nucl. Phys. A 886, 17 (2012) [arXiv:1201.2665 [hep-ph]].

[14] A. R. Zhitnitsky, Nucl. Phys. A 897, 93 (2013) [arXiv:1208.2697 [hep-ph]].

[15] A. R. Zhitnitsky, Phys. Rev. D 89, 063529 (2014) [arXiv:1310.2258 [hep-th]].

[16] A. R. Zhitnitsky, Phys. Rev. D 90, 043504 (2014) [arXiv:1404.5965 [hep-ph]].

[17] E. Witten, Nucl. Phys. B 156, 269 (1979).

[18] G. Veneziano, Nucl. Phys. B 159, 213 (1979).

[19] P. Di Vecchia and G. Veneziano, Nucl. Phys. B 171, 253 (1980).

[20] E. -M. Ilgenfritz, K. Koller, Y. Koma, G. Schierholz, T. Streuer and V. Weinberg, Phys. Rev. D 76, 034506 (2007) [arXiv:0705.0018 [hep-lat]].

[21] E. -M. Ilgenfritz, D. Leinweber, P. Moran, K. Koller, G. Schierholz and V. Weinberg, Phys. Rev. D 77, 074502 (2008) [Erratum-ibid. D 77, 099902 (2008)] [arXiv:0801.1725 [hep-lat]].

[22] F. Bruckmann, F. Gruber, N. Cundy, A. Schafer and T. Lippert, Phys. Lett. B 707, 278 (2012) [arXiv:1107.0897 [hep-lat]].

[23] M. Luscher, Phys. Lett. B 78, 465 (1978).

[24] M. Ünsal and L. G. Yaffe, Phys. Rev. D 78, 065035 (2008). [arXiv:0803.0344 [hep-th]].

[25] E. Thomas and A. R. Zhitnitsky, Phys. Rev. D 85, 044039 (2012) [arXiv:1109.2608 [hep-th]].

[26] A. Bhoonah, E. Thomas and A. R. Zhitnitsky, Nucl. Phys. B xxx, to appear. [arXiv:1407.5121 [hep-ph]].

[27] A. Yamamoto, Phys. Rev. D 90, 054510 (2014) [arXiv:1405.6665 [hep-lat]].

[28] Y. B. Zeldovich, JETP Lett. 6, 316 (1967) [Pisma Zh. Eksp. Teor. Fiz. 6, 883 (1967)].

[29] J. Sola, J. Phys. Conf. Ser. 453, 012015 (2013) [arXiv:1306.1527 [gr-qc]].

[30] A. Guth, Phys. Rev. D 23 (1981) 347;

A. Linde, Phys. Lett. B 108 (1982) 389.

[31] A. D. Linde, Inflationary Cosmology, Lect. Notes Phys. 738, 1 (2008) [arXiv:0705.0164 [hepth]]

[32] V. Mukhanov, Physical Foundation of Cosmology, Cambridge Univ. Pr. , 2005.

[33] C. Cao, M. van Caspel and A. R. Zhitnitsky, Phys. Rev. D 87, no. 10, 105012 (2013) [arXiv:1301.1706 [hep-th]].

[34] A. R. Zhitnitsky, Phys. Rev. D 88, no. 10, 105029 (2013) [arXiv:1308.1960 [hep-th]].

[35] A. Zhitnitsky, Phys. Rev. D 90, no. 10, 105007 (2014) [arXiv:1407.3804 [hep-th]]. 